\title{
WIRELESS SENSOR NETWORK-BASED ACQUISITION AND ANALYSIS OF MECHANICAL VIBRATION SIGNALS
}

\author{
Lingyun Liu ${ }^{1}$ \\ ${ }^{1}$ School of Physics, Jining Teachers College, Jining 012000, China. \\ Email: liulingyunjin168@126.com
}

\begin{abstract}
Objective: To judge the failure of mechanical equipment, a method of collecting and analysing mechanical vibration signals based on Wireless Sensor Network (WSN) is studied. Methods: The WSN-based hardware designs of the vibration signal acquisition node are introduced separately, including six modules of processor, wireless communication, signal conditioning, data acquisition, data storage, and power management. Software design method: Based on the IAR Embedded Workbench (IDE) development environment, the star topology network mode coordinator (COORD) terminal node (END) program set up by ZigBee is designed. The combination of the Empirical Wavelet Transform (EWT) and envelope spectrum in the LabVIEW toolkit processes the collected signal data. Finally, the design method is verified by simulation. Results: The designed node acquisition method can effectively detect mechanical vibration signals; the analysis of signal data shows that the data processing and analysis method based on EWT can accurately divide the vibration signal data. Conclusion: The WSN-based method can accurately divide the vibration signal data, thereby judging the signal of mechanical failure and identifying the type of failure accurately, which provides a theoretical basis for mechanical signal monitoring and mechanical failure maintenance in actual applications.
\end{abstract}

Keywords: WSN; Mechanical Vibration Signal; Wavelet Transform; Coordinator; Terminal Node.

\section{Introduction}

In recent years, with the rapid development of science and technology, modern machinery and equipment have been increasingly applied to various industries. Therefore, the functions, production efficiency, precision, and automation of machinery and equipment have increased constantly. Meanwhile, the problem of reliability and safety of machinery is becoming increasingly prominent [1].

Various engineering machinery and equipment, from space shuttles to small motors, have vibrations. To ensure the reliability of these mechanical structures, the monitoring of mechanical vibration conditions has become an important subject in the field of the engineering technology [2].

A Wireless Sensor Network (WSN) is a distributed sensor network that combines MicroElectro-Mechanism System (MEMS) technology, wireless communication technology, low-power embedded technology, and System on Chip (SoC) technology. It is a novel method of information acquisition, and its tip is a sensor that can sense and examine the external world [3].

The sensors in WSN can communicate wirelessly and can be wired or wirelessly connected to the Internet. It has the characteristics of selforganization, scalability, and convenient installation and deployment [4].
WSN is widely used in fields such as military, intelligent transportation, environmental monitoring, and medicine. WSN also provides innovative idea and method for monitoring mechanical vibration signals. Compared with traditional vibration monitoring systems, it has the advantages of convenient and fast installation, mobile monitoring, and high accuracy of information collection [5].

Many researchers have researched the collection and analysis of mechanical vibration signals by WSN. For example, Saadon and Sidek (2015) proposed a model of an array energy harvester based on WSN MEMS. Through simulation analysis, it was found that micro-generators could become a substitute for battery solutions in the future [6].

To improve the low efficiency of current lossless compression methods for mechanical vibration WSN, Huang et al. (2015) proposed a method for data block lossless compression, which mainly consisted of data segmentation and data encoding [7].

However, WSN has many unsolved problems in the field of mechanical vibration signal monitoring, including energy consumption control, energy supply, and high-speed synchronous acquisition of vibration signal data.

Through the collection of mechanical vibration signals and the analysis of these signal data, the operating status of the mechanical equipment can be 
determined, as well as evaluating the safety and diagnosing the fault of the mechanical equipment [8].

Since the 1960s and 1970s, there have been researches on the fault diagnosis of mechanical equipment in foreign countries. For example, the University of Manchester in the UK has conducted a long-term study on the reliability of online monitoring of mechanical equipment, which has achieved significant results [9].

Although the research on WSN has started late in China, through the efforts of many scholars, China has also made great progress. In the application of some special equipment, a system for detecting the equipment and related sensors is designed. For example, a new non-stationary signal time-frequency analysis method is proposed and applied to the experimental data analysis of rolling bearings, which can effectively diagnose the faults of the rolling bearings [10].

This study collects the mechanical vibration signals through the overall design of the WSN node, analyzes the mechanical vibration signals based on the Empirical Wavelet Transform (EWT) theory, and validates the method in the application of mechanical fault diagnosis. It is proved that the signal acquisition of the WSN-based mechanical vibration signal acquisition node is more accurate and precise.

\section{Methods}

The collection of mechanical vibration signals based on WSN is mainly completed by vibration signal acquisition nodes, routing nodes, gateway nodes, monitoring management software, and other components. First, the acquisition node converts the vibration signals that the sensor captures into digital signals, sends them to the routing node wirelessly, and transmits them to the gateway node.

Finally, the gateway node transmits the vibration signals to the monitoring and management software for analysis and processing to obtain the monitoring results of mechanical vibration.

\subsection{Hardware design of mechanical vibration WSN node}

At present, most of the WSN-based mechanical vibration signal acquisition nodes use the dual-core architecture of MEMS technology. However, due to the weak mechanical vibration signals, wide frequency distribution, and poor multi-point acquisition synchronization, the acquisition accuracy is not high [11].

Therefore, to reduce the coupling between the collection task and the data transmission task during the data collection process, a collection node with a dual-core processor architecture is designed to control the data collection task and the wireless data transmission task according to the actual needs.

The WSN-based mechanical vibration signal acquisition node includes six modules, i.e., processor, wireless communication, signal conditioning, data acquisition, data storage, and power management. The overall architecture of the design node is shown in Figure 1:

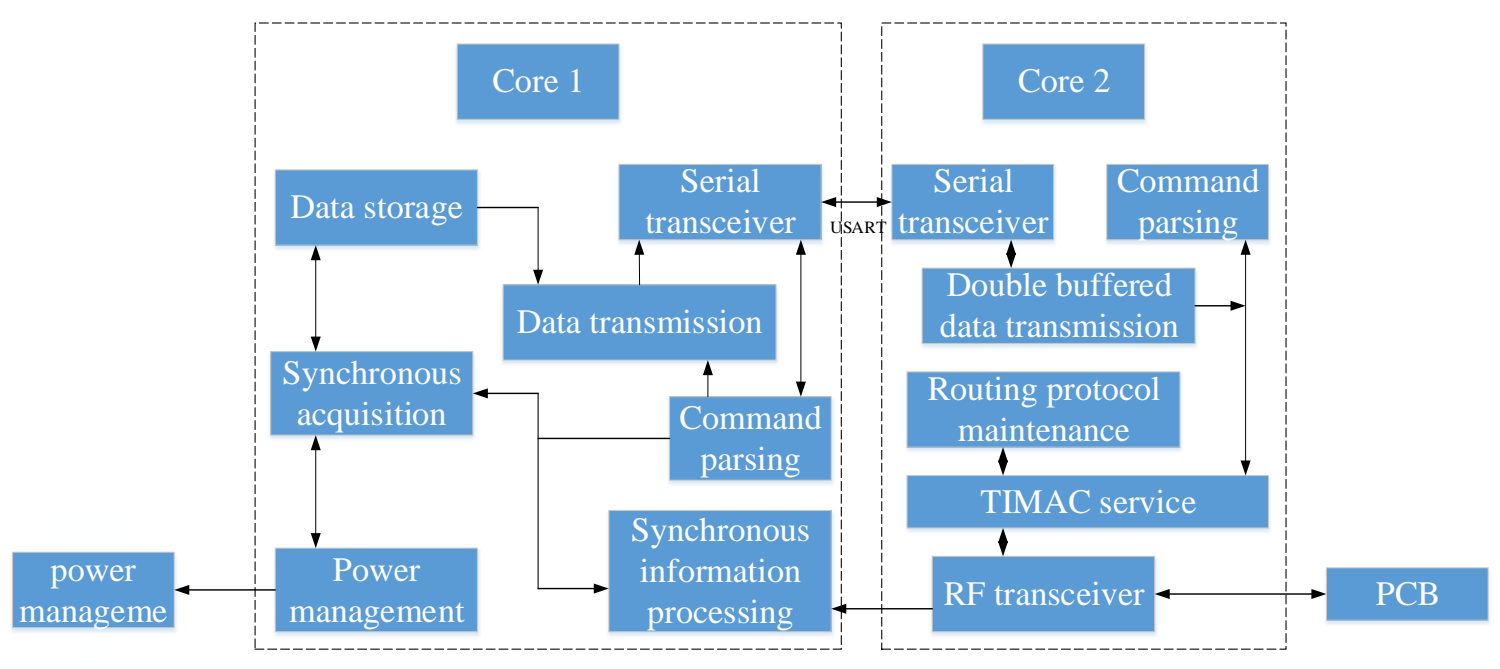

Figure 1: Overall hardware design of WSN signal acquisition

The processor module Core 1 uses the 32-bit microprocessor STM32F405RG of the Cortex ${ }^{\mathrm{TM}}$-M4 core, which can ensure the synchronous data processing with high-precision, sufficient memory space, and abundant external device resources, including general and advanced timers, Universal Synchronous Asynchronous Receiver Transmitter
(USART), Serial Peripheral Interface (SPI), Secure Digital Input and Output Interface (SDIO), Joint Test Action Group (JTAG), and General-Purpose Input and Output interface (GPIO).

The processor module Core 2 uses the CC2530 wireless radio frequency communication chip and also has rich external equipment resources, 
including general-purpose timer, USART, and GPIO Analog-to-Digital (ADC) converter, which is suitable for low power consumption requirements.

The functions of Core 1 and Core 2 are mutually independent. Core 1 is mainly responsible for tasks of data acquisition, storage, and sending. Core 2 is mainly responsible for tasks of network maintenance, command forwarding, and data transmission.

They exchange data through USART. After receiving the network control command, Core 2 forwards the command to Core 1 through USART.
After receiving the control command, Core 1 performs the corresponding data collection, storage, and transmission tasks. Then, it uploads the data to Core 2 through USART. Thus, core 1 can collect highly precise and highly frequent vibration signals without interference from factors such as networks and data transmission.

The wireless communication module uses the MAC-based ZigBee WSN protocol stack.

Figure 2 is a schematic diagram of the protocol stack:

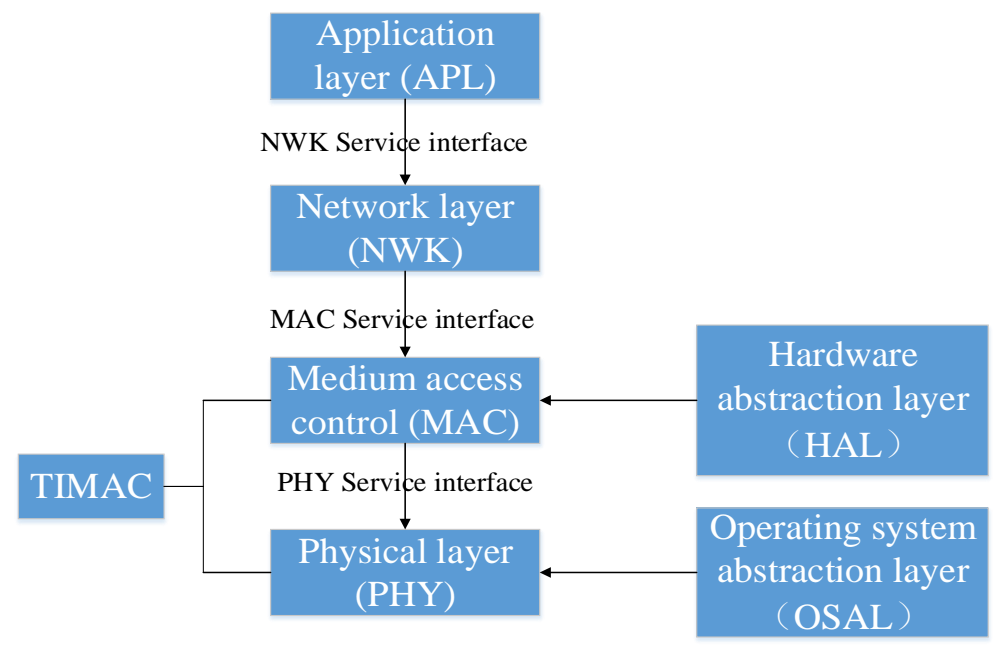

Figure 2: WSN protocol stack

As shown in Figure 2, the ZigBee WSN protocol stack includes the physical layer (PHY), the Medium Access Control (MAC), the network layer (NWK), and the application layer (APL). The data transmission specifications of the PHY layer and the MAC layer are defined by IEEE802.15.4. The data transmission specifications of the NWK layer and the APL layer are defined by the ZigBee protocol.

TIMAC is a variety of wireless radio frequency communication chips that meet the requirements of CC2530 and CC2630, which can be used to quickly build low-power and low-cost wireless communication products [12].

TIMAC can provide an application programming interface for OSAL, HAL, and MAC, which is convenient for programming and controlling the device. Networks can also be formed automatically. Networks established in beacon mode can use periodic beacons to achieve clock synchronization between nodes.

The sensor selects the Integrated Circuits Piezoelectric (ICP) piezoelectric acceleration sensor. This acceleration sensor can meet the requirements of a wide range of acceleration and frequency vibration shock testing. When installing, the sensor should be insulated from the test point to avoid the impacts of ground circuit noise. In the signal conditioning module, the most important step is to amplify and filter the signal collected by the selected sensor before transmission, mainly including three aspects, i.e., the current source design that can provide a stable power supply for the sensor, the programmable gain amplifier module that amplifies the signals collected by the sensors, and the signal filtering module that performs filtering operations on the signal data. Finally, the signal data is transmitted to an Analog-to-Digital (A/D) conversion module for A/D conversion.

When selecting the A/D converter for the data acquisition module, it is necessary to select the ADS8433 with a higher data sampling rate and resolution. Its sampling rate can reach $100 \mathrm{kHz}$, the resolution is 16 bits, the signal-to-noise ratio is 89 , and the power consumption is low.

The advantages of multiplexed and differential inputs are ideal for battery-powered systems.

To ensure the high accuracy of the A/D conversion result, a low-power precision reference voltage source REF3130 is used to provide a reference voltage for the A/D converter. Its output voltage is $3.0 \mathrm{~V}$ with an error as low as $0.2 \%$.

The data collected by the data acquisition module need to be stored first. Then, the data are uploaded to the system for analysis and processing.

Therefore, a suitable storage unit needs to be selected in the data storage module. Currently, the most commonly used data storage module is NAND Flash, which has a faster reading and writing speed. 
It is the main component of the SD cards and flash disks. Therefore, this study uses the SD card as the node storage device and the SDIO interface for data transmission. The node SDIO controller reads and writes the SD card in 4-bit mode, and the data exchange rate can reach up to $10 \mathrm{MB} / \mathrm{s}$, which fully meets the requirements for high-rate data storage.

The power management module needs a highquality power supply system.

This study uses two different power supply methods. The terminal nodes are generally batterypowered and easy to move, while the coordinator nodes are generally powered by USB interfaces. Since the main chips of this node, such as the microprocessor, ZigBee transmission module, FLASH memory, and data acquisition chip, have a working voltage of $+3.3 \mathrm{~V}$, the power supply voltage of the filter module and constant current source chip needs $+5 \mathrm{~V}$ and $+15 \mathrm{~V}$, respectively. In addition, the power supply voltage of the node power supply needs to be $+3.3 \mathrm{~V},+5 \mathrm{~V}$, and $+15 \mathrm{~V}$. A boost converter or voltage regulator can be selected for voltage conversion.

\subsection{Software design of mechanical vibration WSN node}

The software development environment of the WSN node is IAR Embedded Workbench (EW). EW can provide a very intuitive working interface, supporting 8-bit, 16-bit, 32-bit microprocessors. It integrates an embedded $\mathrm{C} / \mathrm{C}++$ optimized compiler, assembly tool, linker, text editor, and CSPY debugger.

The EW is simple, convenient, and powerful, which brings great convenience to the development and debugging of data collection nodes [13].

The star topology network mode formed by ZigBee is composed of a main coordinator (COORD) and multiple terminal nodes (END). This mode is easy to manage and maintain.

The structure is shown in Figure 3:

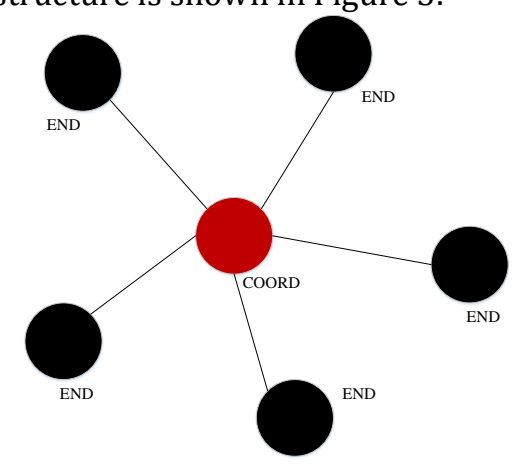

Figure 3: Star topology structure

According to the network mode, this study chooses to design the terminal node as a point-topoint mode, and the coordinator node is designed as a one-to-many mode. The network is automatically completed by setting two I0 ports, i.e., M0.0 and M0.1, to complete the networking. The transmission module only needs to set the private network ID number, serial port baud rate, transmit power, communication channel, periodic wake-up frequency of the terminal device, point-to-point mode backup address, and restarting command.

The commands are respectively PANID, BAUD_RATE, TX_POWER, CHANNEL, POLL_RATE, UNI_SEC_ADDR, and PW_RESET [14].

The terminal node is added to the coordinator node through these parameters of the transmission module, as shown in Figure 4 and Figure 5, respectively, the flowchart of the coordinator node and terminal node program designed in this study.

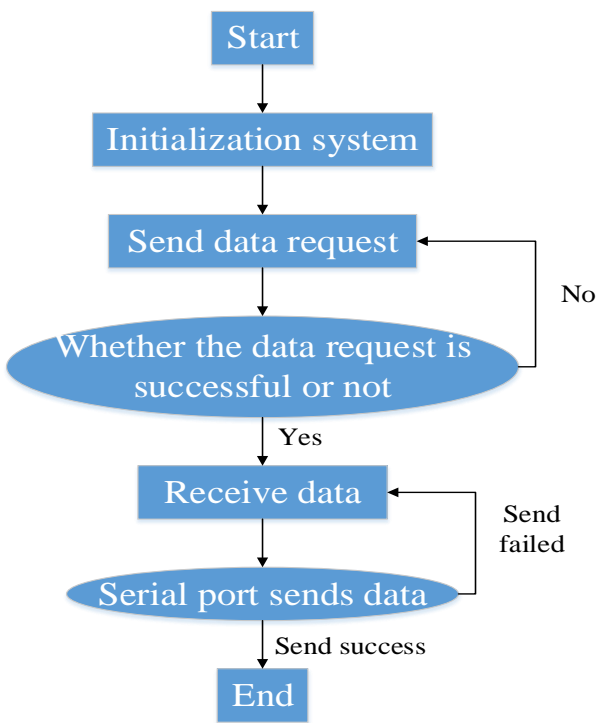

Figure 4: Workflow of coordinator node program

As shown in Figure 4, the main function of the coordinator node is to establish a network, collect the mechanical vibration signal sent from the terminal node, and transmit the signal to the monitoring system through the serial port. After the power is turned on, the coordinator enters the running state, and the system will automatically complete the initialization.

This process mainly includes the initialization of serial ports, I/O ports, and ZigBee serial transmission modules. Then, the network will be automatically networked according to the set parameters. After the network is set up, since the data is not received in real-time, it is necessary to first determine whether there is a data transmission request before the data is sent.

When the terminal node collects a signal at a specified time, it will send a data transmission request, and the coordinator node will enter the receiving operation state according to the request and judge the received data at the same time. If the wireless signal data transmitted from the terminal node is received, the received data is saved and transmitted to the upper computer through the serial port. If the monitoring system receives successfully, the memory occupied by the data is released; otherwise, it needs to be resent. 


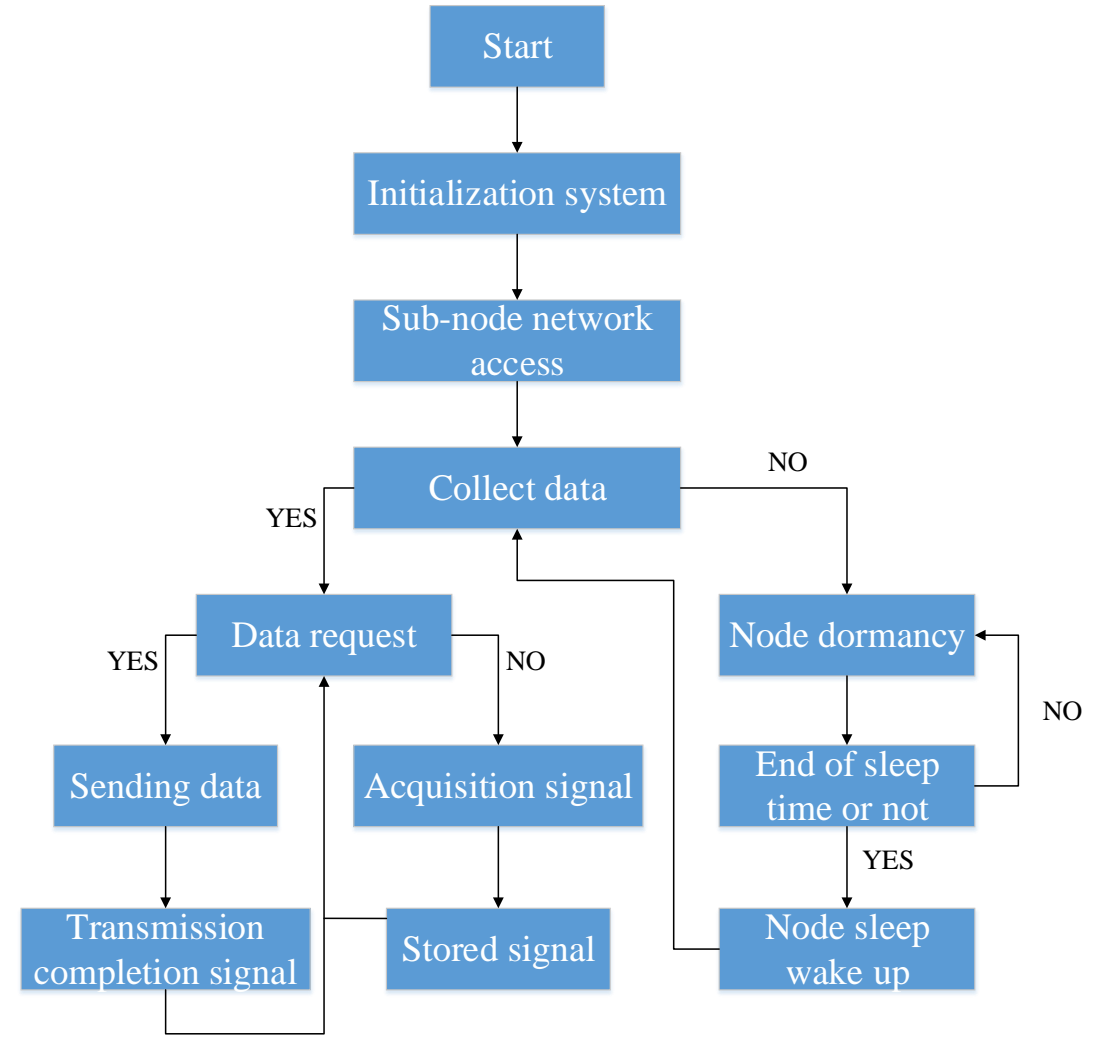

Figure 5: Workflow of terminal node program

As shown in Figure 5, the program design of the terminal node includes the initialization system program, data acquisition program, data transmission, and stop acquisition/sending tasks. Initializing the system is mainly to set the processor, including powering on the chip, defining each I0 interface, and variables. The data acquisition subroutine is mainly used to read and A/D33convert the signal collected by the sensor. Data transmission is to send data from the storage area to the sending area through the SPI port. After being processed, these signal data are sent to the coordinator. When the terminal node does not collect and send tasks, it will automatically enter the sleeping mode and will wake up when waiting for tasks. By connecting the sensors to the wired data acquisition system NI9234 and WSN nodes, the designed node signal acquisition method is compared with the wired signal acquisition method.

\subsection{Signal analysis based on EWT algorithm}

After the WSN node transmits the collected mechanical vibration signal data to the monitoring system, it is necessary to analyze the data to determine whether the machine has failed.

The mechanical vibration signal data is nonstationary and non-linear. Therefore, to make the result more accurate, this study uses the wavelet transform algorithm to analyze the signal data.

The vibration signal is decomposed into local basic quantities from the time and frequency domain. Then, the signal is submitted to targeted analysis.

EWT is currently the most widely used adaptive processing method. Its essence is to perform a Fast Fourier transform (FFT) on the original signal, divide the obtained Fourier spectrum, and then establish and process a set of suitable wavelet filter banks of each divided Fourier spectrum [15].

First, FFT processing is performed on the collected signals; then, spectrum processing is performed on the processed signals.

The divided spectrum range is assumed to be [0, $\mathrm{x}$, and the signal is assumed to be $\mathrm{N}$ single component structures. Thus, all the maximum values in the spectrogram are found, and then these maximum values are sorted from the greatest to the least. If the total number of maximum values is $U$, there will be two cases:

(1) When $U \geq N$, there are enough maximum values to provide the boundary required for segmentation, and only the first $\mathrm{N}-1$ maximum value is retained;

(2) When $U<N$, because there are not enough maximum points, all the points can be retained and reassigned to $\mathrm{N}$.

Figure 6 is a schematic diagram of spectrum division. Each interval after spectrum division is assumed to be $\mathrm{Q} n=[\mathrm{An}-1, \mathrm{An}], \mathrm{n}=1,2, \ldots, \mathrm{N}$, and $\mathrm{a}$ transition period $\mathrm{Tn}$ is defined around each $\mathrm{Q}$.

This requires $\mathrm{N}+1$ boundaries.

In addition to the known 0 and $\mathrm{x}$ boundaries, $\mathrm{N}-1$ boundaries are needed. 


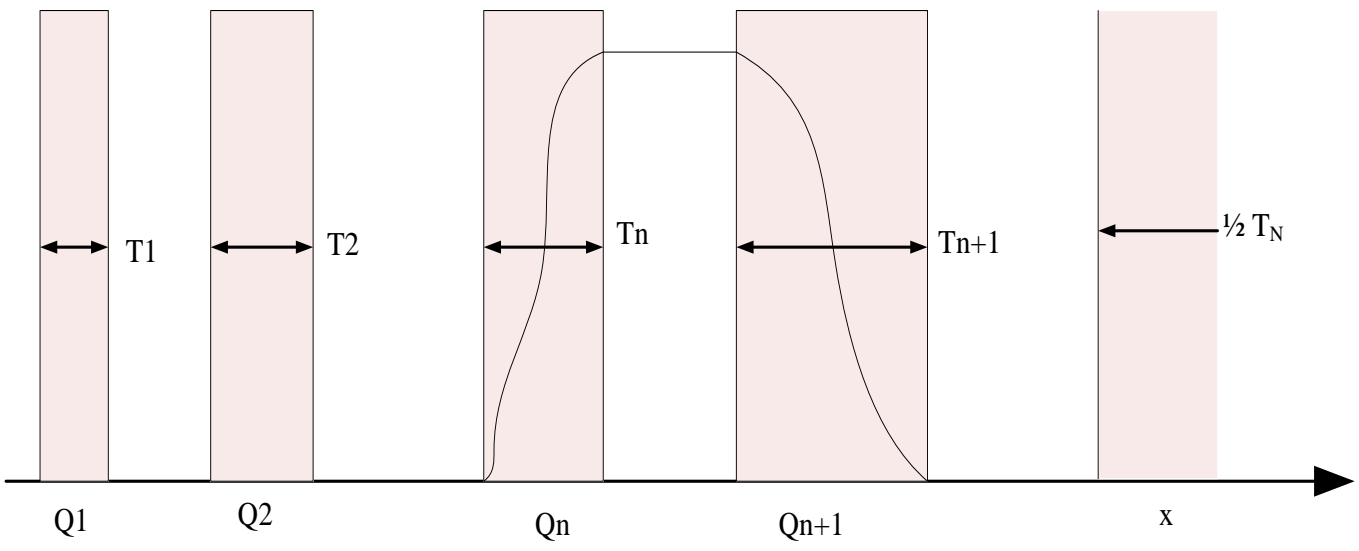

Figure 6: Schematic diagram of spectrum division

After dividing each spectrum $Q$, the data is processed and analyzed by constructing a wavelet transform function. The EWT function is established according to the classic wavelet transform, which can obtain:

$Q_{f}^{g}(0, t)=<f, \Psi_{n}>=\int f(T) \overline{\Psi_{n}(T-t)} d T=$

\section{$\left[\bar{f}(Q) \overline{\overline{\Psi_{n}}}(Q)\right]$}

$Q_{f}^{g}(0, t)=<f, \Phi_{1}>=\int f(T) \overline{\Phi_{1}(T-t)} d T=$

\section{$\left[\bar{f}(Q) \overline{\left.\overline{\phi_{1}}(Q)\right]}\right.$}

In the above equations, $\overline{\Psi_{n}}(Q)$ and $\overline{\Phi_{1}}(Q)$ are the Fourier Transform functions of $\Psi_{\text {n }}$ and $\Phi_{1}$, respectively; $\overline{\Psi_{n}(T-t)}$ and $\overline{\Phi_{1}(T-t)}$ are the complex conjugate functions of $\Psi_{n}$ and $\Phi_{1}$, respectively; <,> is the inner product calculation. Then, the empirical mode functions can be expressed as:

$$
\begin{aligned}
& f_{0}(t)=Q_{f}^{\sigma}(0, t) * \Phi_{1}(t) \\
& f_{n}(t)=Q_{f}^{\sigma}(n, t) * \Psi_{n}(t)
\end{aligned}
$$

In above equations, * is the convolution.
To verify the practicability of the EWT algorithm in mechanical signal analysis, the above functions are combined with the envelope spectrum in the LabVIEW toolkit for simulation analysis.

Finally, the WSN node designed in this study is tested on a commonly used mechanical fault simulation platform (MFS-MG).

\section{Results \\ 3.1 Data acquisition results}

To verify the signal acquisition effect of the WSNbased mechanical vibration signal node designed in this study, an analog acquisition node is set up according to the method in this study. For convenience, this study only builds a terminal and a coordinator. After the setup is completed, the terminal node switch is turned on. At this time, the node indicator is always on, and it will be off when the collection is completed. This is also in line with the low-power design.

After the collected data is transmitted to the monitoring system, the waveform figure of the vibration signals can be obtained.

Table 1 shows the comparison of the main spectral lines of the mechanical vibration signal acquired by the WSN node acquisition method and the wired signal acquisition method.

Table 1.Comparison of main spectral lines

\begin{tabular}{|c|c|c|c|c|c|}
\hline \multicolumn{3}{|c|}{ Frequency /HZ } & \multicolumn{3}{c|}{ Amplitude /mV } \\
\hline Node & NI9234 & Error /\% & Node & NI9234 & Error /\% \\
\hline 1350 & 1350 & 0 & 1.586 & 1.557 & 1.83 \\
\hline 3355 & 3355 & 0 & 2.459 & 2.430 & 1.18 \\
\hline 6215 & 6215 & 0 & 0.987 & 0.962 & 2.53 \\
\hline
\end{tabular}

As shown in Table 1, the nodes designed in this study can collect mechanical vibration signals. In addition, the collected signal data have higher accuracy, and the acquisition results of the designed method are better than those of the wired method, which provides a basis for the analysis of signal data. 


\subsection{Simulation experiment results}

This study designs the hardware and software of the mechanical vibration signal acquisition node based on WSN to collect the vibration signal data and analyzes the data through the EWT algorithm. Figure 7 shows the simulation results after EWT Fourier spectrum division:

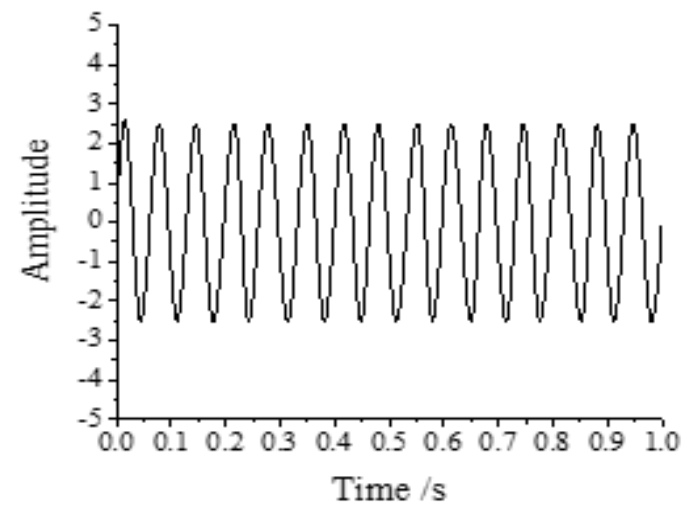

a. Sinusoidal signal

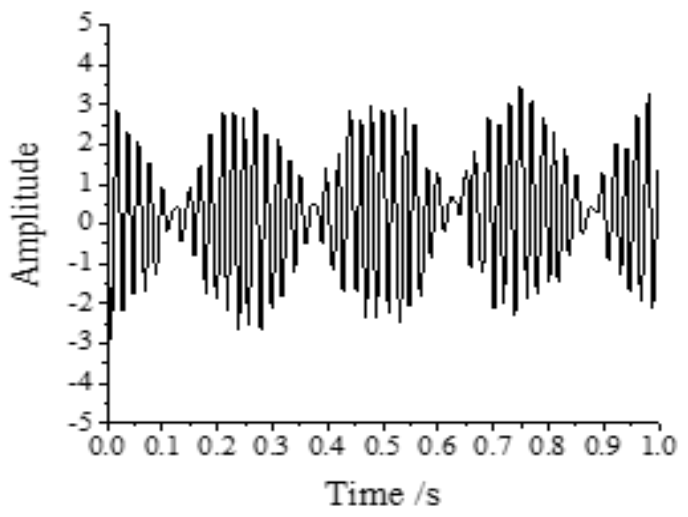

\section{b. Amplitude modulated signal}

Figure 7: EWT simulation results

As shown in Figure 7, a and b respectively represent the different signals of the two different components after the Fourier spectrum division.

Apparently, $a$ is a sinusoidal signal and $b$ is an AM signal. This shows that the EWT algorithm used in this study can well divide the vibration signals.

\subsection{Data analysis results}

The design method of this study is tested on the MFS-MG mechanical fault simulation verification platform. The mechanical vibration signals are collected; then, the collected signals are decomposed and analyzed by the combination of the EWT algorithm and the envelope spectrum to obtain the above $\mathrm{a}$ and $\mathrm{b}$ components.
The results of the envelope spectrum analysis are shown in Figure 8.

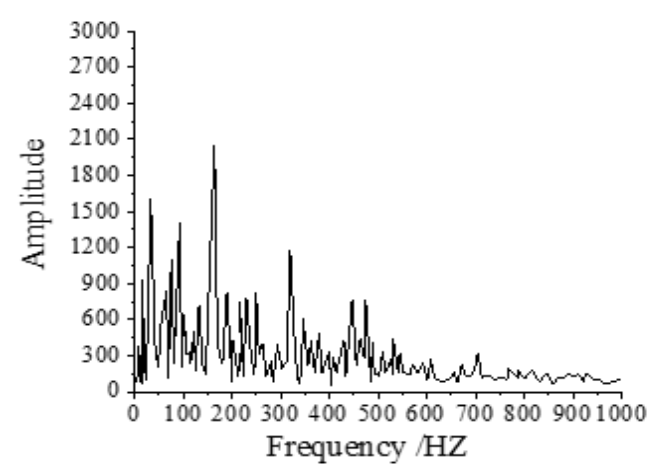

Spectral component a

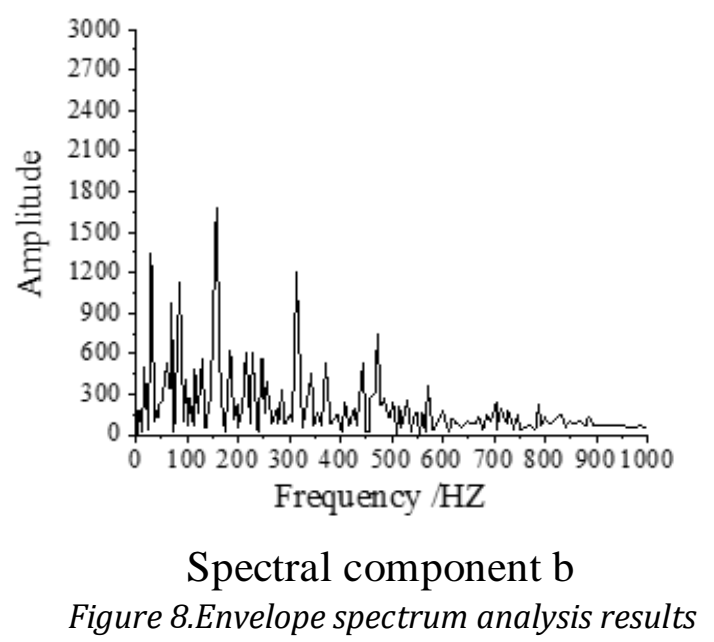

As shown in Figure 8, the comparison of the waveform diagrams shows that the waveform of the b component is more obvious than that of a component.

Also, the amplitude of the vibration signal of $b$ is more obvious.

In addition, the characteristic frequency, fundamental frequency, and their corresponding octave and side spectral lines can be found in the spectrogram.

Besides, the amplitude of the b component is low, indicating that the filtering effect is the most obvious and a fault frequency exists at the frequency.

According to the simulation results, after analyzing and processing with the combination of EWT algorithm and envelope spectrum, the WSNbased mechanical vibration signal acquisition node designed and proposed in this study can accurately divide the vibration signal data, as well as precisely judge the mechanical failures and identify the types of failures from the signal data. 


\section{Conclusions}

This study introduces the hardware and software design methods of WSN nodes in detail and verifies the design results.

The results show that the design method of this study can effectively collect mechanical vibration signals.

The EWT algorithm is used to decompose and analyze the collected signal data.

The method can accurately detect the mechanical fault signals. Also, the types of mechanical faults can be clarified based on data analysis.

The proposed method can be applied to mechanical signal monitoring and mechanical fault repairing, which provides a theoretical basis to improve the safety performance and service life of the machine. However, there are still some shortcomings in this study. For example, the dualcore processor consumes a large amount of power; thus, the system cannot monitor the machinery for a long time. In the subsequent study, reducing energy consumption can be the focus.

\section{References}

[1] L. X.Quan, S.Liu, Z. X.Jiao, et al.,"Mechanicalvibration transfer path analysis of swash-plate axial piston pump back shell,"Journal of Vibration Engineering, vol.30, no.4, pp. 587-595, 2017.

[2] H. Yang, A. Benjamin, B. Anthony,"Simulation of multi-source external mechanical vibration for shear wave elastography,"Acoustical Society of America Journal, vol.142, no.4, pp. 2628-2628, 2017.

[3] M. X. Dong, K.Ota, A. F. Liu,"RMER: Reliable and Energy-Efficient Data Collection for Large-Scale Wireless Sensor Networks,"IEEE Internet of Things Journal, vol.3, no.4, pp. 511-519, 2017.

[4] V. C.Güngör, G. P.Hancke, "Industrial Wireless Sensor Networks: Applications, Protocols, and Standards," CRC Press, 2017.

[5] F.Zhu, J. F. Wei, "Localization Algorithm in Wireless Sensor Networks Based on Improved Support Vector Machine,"Journal of Nanoelectronics \& Optoelectronics, vol.12, no.5, pp. 452-459, 2017.

[6] S.Saadon, O. Sidek, "Micro-Electro-Mechanical System (MEMS) - Based Piezoelectric Energy
Harvester for Ambient Vibrations,"Procedia Social and Behavioral Sciences, vol.195, pp. 2353-2362, 2015.

[7] Q. Q.Huang, B. P. Tang, L. Deng, et al.,"Data block-based lossless compression for machine vibration wireless sensor networks," Chinese Journal of Scientific Instrument,vol.36, no.7, pp. 1605-1610,2015.

A. K. Batra, A.Alomari, A. K.Chilvery, et al.,"Piezoelectric Power Harvesting Devices: An Overview,"Advanced Science, vol.8, no.1, pp. 112,2016.

[8] E.Farjah, H.Givi, T.Ghanbari,"Application of an Efficient Rogowski Coil Sensor for Switch Fault Diagnosis and Capacitor ESR Monitoring in Nonisolated Single-Switch DC-DC Converters,"IEEE Transactions on Power Electronics,vol.32, no.2, pp. 1442-1456, 2017.

[9] J. D. Zheng, H. Y.Pan, X. L. Qi, et al.,"Enhanced Empirical Wavelet Transform Based TimeFrequency Analysis and Its Application to Rolling Bearing Fault Diagnosis," Tien Tzu Hsueh Pao/acta Electronica Sinica,vol. 46, no.2, pp. 358-364, 2018.

[10] J. Li, M.Li, J.Zhang,"Rolling bearing fault diagnosis based on time-delayed feedback monostable stochastic resonance and adaptive minimum entropy deconvolution,"Journal of Sound Vibration,vol.401, pp. 139-151, 2015.

[11] J. G.Wu, H. D. Shi, T. L. Zhao, et al.,"Hightemperature BiSc03-PbTiO3 Piezoelectric Vibration Energy Harvester," Advanced Functional Materials, vol.26, no.39, pp. 71867194, 2016.

[12] J. Liu, O. F.Beyca, P. K. Rao, et al.,"Dirichlet Process Gaussian Mixture (DPGM) Models for Real-Time Monitoring and its Application to Chemical Mechanical Planarization (CMP),"IEEE Transactions on Automation Science \& Engineering, vol.99, pp. 208-221, 2016.

[13] S.Siddheswar, S.Biplab, D.Uma,"Design of Wireless Sensor Node to Measure Vibration and Environment Parameter for Structural Health Monitoring Application,"Advances in Intelligent Systems and Computing, vol.343, pp. 59-65, 2015.

[14] C. Jiang, X. Zhou, "Application of laser selfmixing interference technology and wavelet transform in gearbox fault diagnosis," Optical Technique, vol.43, no.1, pp. 83-86, 2017. 